\title{
Research on Reasoning Logic in Mongolian Medicine
}

\author{
Xiaohui Wang ${ }^{1}$, Na Yi ${ }^{2}$, Siwen Wei ${ }^{2}$, Lidao Bao ${ }^{1,2, ~ * ~}$ \\ ${ }^{1}$ College of Pharmacy, Inner Mongolia Medical University, Hohhot, China \\ ${ }^{2}$ Department of Pharmacy, Affiliated Hospital of Inner Mongolia Medical University, Hohhot, China
}

\section{Email address:}

349881704@qq.com (Xiaohui Wang), baolidao237@qq.com (Lidao Bao)

${ }^{*}$ Corresponding author

\section{To cite this article:}

Xiaohui Wang, Na Yi, Siwen Wei, Lidao Bao. Research on Reasoning Logic in Mongolian Medicine. Humanities and Social Sciences. Vol. 5, No. 1, 2017, pp. 9-13. doi: 10.11648/j.hss.20170501.13

Received: December 8, 2016; Accepted: January 20, 2017; Published: February 10, 2017

\begin{abstract}
Mongolian medicine is one of the basic disciplines of Mongolian Studies. Mongolian medicine plays an important role in prevention and treatment of diseases in northern ethnic minorities, particularly the Mongol nationality. As an important research field of Mongolian Studies and an important part of the traditional Mongolian culture, Mongolian medicine has its own unique position. Nowadays, Mongolian medicine is the mainstream medicine in the minority areas due to its uniqueness and practicability and its inheritance depending on scientificity during development and improvement of the medicine. In addition, it stands out from various types of medicine in the minority areas and is generally accepted by the public. Thus, it is arousing attention from the domestic and overseas medical circles and the relevant professionals. The long history of Mongolian medicine is highlighting the important of the logical thinking method. The Mongolian medicine makes great contribution to medicine. The thinking mode and reasoning method of Mongolian medicine play a notably important role in diagnosis and treatment of various diseases. The paper aims to explore the thinking and reasoning connotations of Mongolian medicine. The paper preliminarily investigates the significance of logic in Mongolian medicine, the theoretical system and logic thinking in Mongolian medicine, dynamic treatment and comprehensive reasoning in Mongolian medicine.
\end{abstract}

Keywords: Mongolian Medicine, Logic Thinking, Reasoning

\section{Background}

Mongolian medicine is short for traditional Mongolian medicine. Mongolian medicine is one of the basic disciplines of Mongolian Studies, a result of the long-term production and wisdom, and an important part of the traditional culture of the Mongol nationality [1]. Nowadays, Mongolian medicine is the mainstream medicine in the minority areas due to its uniqueness and practicability and its inheritance depending on scientificity during development and improvement of the medicine. In addition, it stands out from various types of medicine in the minority areas and is generally accepted by the public. Thus, it is arousing attention from the domestic and overseas medical circles and the relevant professionals [2].

The long history of Mongolian medicine is highlighting the important of the logical thinking method. What are the main characteristics of the logic thinking in Mongolian medicine? What is the reasoning process of diagnosis and treatment in Mongolian medicine? These questions deeply inspire us and they are also the background of this paper.

The research on the reasoning method in Mongolian medicine can improve the teaching quality of Mongolian medicine and help cultivate more high-quality Mongolian medicine talents. It can also help the medical workers to improve the diagnostic accuracy thus significantly improving the recovery rate in patients. In addition, it can show the diagnostic and treatment thinking in a clearer manner and seek a scientific method for research and improvement of Mongolian medicine.

\section{Research Status of Western Logic Medicine}

Since 1980s, UNESCO (united nations educational scientific and cultural organization) has specified seven major basic disciplines. As a basic discipline, logic is also included. Currently, the research on logic is developing 
rapidly and showing its roles in many aspects under the joint efforts of the domestic and overseas scholars. The logic can serve as a tool in various disciplines and its internal essential characteristics are shown in many disciplines [3].

Western medicine is the leader of the modern medicine. The research on any type of medicine necessarily involves the contrast and reference to the western medicine. The development of western medicine is based on scientific development. The scientific attitude in the past was speculation of future depending on various phenomena. A typical type of such logic thinking is inductive and deductive reasoning. This method is generally used in scientific verification [4]. However, the biggest problem of such positive thinking is that all developments will limited by the existing facts and it is impossible to predict many unknown future events. Particularly, rapid and timely responses in automation and artificial intelligence are critical. More predictions (expectations) can reduce the response (preparation) time. Thus, the events can be handled immediately after occurrence. Abductive reasoning is the reverse of deductive reasoning. The conclusion can be derived by establishing the fact that the causes of an event is the premise of the result of an event. In other words, abductive reasoning is a process for explaining the known things and a backward exploration method [5]. The conclusion derived from explanations (also a form of prediction) will be much more reliable than that derived from the limited existing data. Such a mode of thinking has been replacing the traditional scientific method [6].

\section{Logic in Mongolian Medicine}

\subsection{Research Status of Logic Medicine of Mongol Nationality}

Medical logic is short for medical logic science. In recent years, such journals as China Medical Management, Medicine and Philosophy, Dialectics of Nature etc. have been publishing articles to discuss the issues regarding medical logic and medical thinking. China's Research Institute of Natural Dialectics, China's Logic Society, and Medicine and Philosophy periodical office have conducted several colloquiums to discuss such problems [7]. Its academic characteristics has evolved from easy to profound, from traditional to modern, from universal to improved, and from scattered to systematic. It exploits a wide prospect for further theoretical research on medical methodology.

Agula presents characteristic traditional therapies in his book titled traditional therapies in Mongolian medicine. The traditional therapies in Mongolian medicine, i.e. the traditional external treatments in Mongolian medicine, are important components of Mongolian medicine. They are various external treatments explored, summarized, studied, and invented during treatment of common diseases, frequently-occurring diseases, and difficult and complicated diseases over the past several thousands of years. It is a discipline that illustrates the principle and involves its application in treatment of diseases [8].

Mongolian medicine has special efficacious diagnostic and treatment methods for multiple diseases such as chronic diseases including digestion, liver and gallbladder, respiration, urinary system, cerebrovascular accidents etc. and rheumatism [9]. It has unique therapeutic effect in respiratory tract infection and digestive system diseases in pediatrics and functional uterine bleeding, uterine fibroid, postpartum diseases in gynecology [10]. Mongolian medicine osteopathy is a unique therapy with significant efficacy in treatment of various bone fractures, bone diseases, and joint diseases. The traditional external therapy in Mongolian medicine is praised as highly efficacious, particularly the method of "treating concussion with concussion". The therapy involving re-concussion of the organs like brain etc. that has been suffered from concussion achieves an astonishingly high recovery rate. The medications used in Mongolian medicine are special. The prescriptions are unique and the medications used are natural. Their advantages and efficacy are significantly superior to those of the chemical drugs. Thus, Mongolian medicine is increasingly enjoying popularity among the public [11].

An Guanbu and Jin $\mathrm{Yu}$ in their Overview of Mongolian Medicine reported that there were numerous medical talents in Qing dynasty. A dozen famous Mongolian medicine experts including Luobusang Danjinzhalacang (1639-1704), Yixibalazhuer (1704-1788), Luobusang Chulurumu (1740-1810), Zhanbula (1789-1838), Zhanbula Daoerji (?-1855) et al. published a dozen medical works including Explanation of Medicine, Detailed Explanation of Disease Types, Four Volumes of Ganlu, Dosimetry of Mongolian Medicine, Selections of Mongolian Medicines, Identification of Mongolian Medicines etc. These works lay a solid foundation for formation and development of modern Mongolian medicine [12].

Ba Jigemude reported in his Brief History of Mongolian Medicine that We also assimilated the cultural and scientific knowledge from our adjacent nationalities in the sixteenth century with the social development and the further development in economy and culture. The economy was dominated by nomadism. Some Mongolian regions on the frontier also began to operate semi-farm semi-pasturing economy [13]. We inherited and developed the traditional cultural heritage. Meanwhile, we were also influenced by the culture of the adjacent nationalities. Historical, literature, and natural scientific works were created thus enriching the ethnic culture. With the development of economy and culture and the further development of traditional medical theories and experience of Mongol nationality, we also substantially assimilated the theories and experience from the Tibetan nationality, Han nationality and so on and the Indian medicine to develop modern Mongolian medicine with systematic theories, rich experience, and unique characteristics [14].

\subsection{Research Thought of Mongolian Medicine Logic}

As an important part of the medicine of ethnic minorities, 
Mongolian medicine has its own long history and unique style. The thinking mode and reasoning method in Mongolian medicine involve analysis of the patients' daily activities, symptoms, and possible causes in combination with the unique theoretical system of Mongolian medicine under the macroscopic vision of harmonious existence of humans in the nature and balance and unity of human internal functions. In Mongolian medicine, the unique ideology of unity of man and universe and the diagnostic and treatment cases in the Mongolian regions are utilized for induction and summarization. The three ways of diagnosis of "look, question and feel the pulse" are also used to diagnose and treat disease $[15,16]$. As one of the organisms living in the nature, humans are closely associated with the nature. Thus, humans should not be separated from the nature. On the contrary, we should analyze the potential causes of diseases with holistic thinking [17]. Humans serve as a unity of various organs of the body. Each organ should not be analyzed separately. On the contrary, diagnosis and treatment should be performed in a macroscopic manner. Various organs perform their own functions and are closely associated. The physicians should have excellent medical skills, have a good command of the medical theories, and understand the climatic characteristics, natural environment, traditional culture etc. in various regions of Mongol nationality thus investigating the causes of diseases from various aspects [18]. It is particularly important to study the characteristics of the thinking mode and the validity of the reasoning method of Mongolian medicine. It is the scientific basis of Mongolian medicine. In addition, there is little research on this aspect. Therefore, it deserves our exploration. Mongolian medicine should also keep up the pace of the times. It is no longer a type of narrow and traditional medicine in the remote region [19].

\subsection{Research Methods for Mongolian Medicine Logic}

Firstly, the literature method. The literature method involves collection of a number of books and materials related to Mongolian medicine logic. However, there are few material regarding this aspect. Thus, we have to extract, process, and summarize opinions from classical works related to Mongolian medicine. It is necessary to pay a visit to the famous elderly Mongolian medicine physicians and document their experience and the summarized diagnostic and treatment methods as the arguments for demonstration in the paper.

Secondly, the comparative synthetic method. The experience and viewpoints of the famous elderly Mongolian medicine physicians are synthetically compared to identify the commonalities of the thinking modes and reasoning methods in Mongolian medicine and form our own opinions. Then, their differences are compared and analyzed on the basis of the climatic and cultural characteristics [21].

Thirdly, combination of theory and practice. The Mongolian medicine physicians and nurses are interviewed and investigated to demonstrate our own opinions in combination with our own viewpoints. We will observe their diagnostic and treatment processes and conduct some questionnaire surveys.

\subsection{Thinking Mode in Mongolian Medicine}

The methodological system of rational knowledge during establishment of the theoretical system of Mongolian medicine is the logic thinking in Mongolian medicine. The essential relationships between the internal and external factors and the regularities are reflected using such thinking modes as concept, judgment, reasoning, etc. [22]. Based on the long-term medical practical activities, Mongolian medicine uses the ancient philosophical thoughts and methodologies, ancient astronomical, geological, climatic, biological, physical, and psychological knowledge to summarize, analyze, and induce the human organization structure, physiological functions, causes, incidence, pathogenesis, health maintenance, diagnosis and treatment etc. It is formed on the basis of multiple cycles of practice-cognition-re-practice-recognition. The thinking mode in Mongolian medicine plays a decisive role in establishing the theoretical system of Mongolian medicine [23].

On the basis of the practice activities in Mongolian medicine and under the guidance of materialism and dynamic thought (idea of balance), the logic thinking in Mongolian medicine uses specific thinking methods including analogy, deduction, balancing, reasoning etc. to rationally understand, induce, and summarize the normalities and abnormalities in human vital activities and the processes of maintaining normalities and correcting the abnormalities [24]. Therefore, the establishment of the theoretical system of Mongolian medicine is the result of integration of the rich experience and philosophical thinking accumulated in the clinical practice of Mongolian medicine. Based on the ancient philosophical thinking and principle, Mongolian medicine raises substantial perceptual materials derived from the experience and observation in medical practical activities to conceptual knowledge. The holistic view, dynamic thought, outlook on life, view of pathogenesis, treatment of diseases etc. in Mongolian medicine focus on the idea of balance [25]. The idea of balance influences Mongolian medicine throughout. This is an inevitable choice instead of an external influence. The practice of Mongolian medicine demonstrates that the ideal of balance not only plays an important role in establishing the theoretical system of Mongolian medicine and but also has clinical significance in guiding health maintenance and disease prevention and medication in diagnosis and treatment [26].

\subsection{Basis and Treatment Methods of Mongolian Medicine Logic}

The dynamic treatment in Mongolian medicine considers the patients as a whole. Based on the reactions in various aspects, Mongolian medicine focuses on both the causes and the stages of disease progression. Also, Mongolian medicine focuses on both the diseases and the differences in physique 
among patients. In Mongolian medicine, the clinical data are collected and analyzed with various diagnostic methods including "look, question and feel the pulse" to obtain the basis for dynamic treatment [27] Based on the complex clinical processes, a series of comprehensive analyses are conducted to identify the major causes of diseases. For example, a patient with lumbago is accompanied with exacerbated pain in cold weather and another patient with lumbago is accompanied with headache, tinnitus, and weakness of limbs. Both patients have the symptoms of lumbago but the major cause of the disease of the former is rheumatism and the major cause of the disease of the latter is deficiency of the kidney. Based on the causes of diseases, different therapeutic principles are established to solve the major contradictions and eliminate the lumbago symptoms, which reflects the dialectical overall unity and flexibility in Mongolian medicine [28]. In Mongolian medicine, the solar terms, weather, geographical conditions, and human factors should be considered. The Mongolian medicine dialectic is a logical reasoning process. Such information as symptoms, signs, etc. is judged and classified synthetically for further reasoning and demonstration. Accurate dynamic treatment requires a comprehensive and deep understanding of such knowledge as the basic theory of Mongolian medicine, diagnostic and logical thinking methods in Mongolian medicine. Such that, the symptoms manifested by the patients can be organically classified, analyzed, and reasoned for identify the treatment orientation [29].

The spread of Mongolian medicine shows its unique reasoning thinking methods and reasoning methods. The logical thinking mode in Mongolian medicine implements the macroscopic examination and whole equilibrium of "unity of humans and nature", "unity of humans and society", and "unity of various components of humans" and utilizes the unique "three-state thinking" for diagnosis and treatment based on an in-depth and thorough understanding of the basic theories of Mongolian medicine [30]. This thinking mode requires solid knowledge in Mongolian medicine, rich clinical experience, the holistic view of considering humans as an integral part of the nature and society, a good understanding and analysis of the internal balance of "Sangen" to reduce the error rate of diagnoses and improve the efficiency of treatment. The reasoning methods in Mongolian medicine are combinations of deductive reasoning, analogic reasoning, inductive reasoning, and abductive reasoning. As ancient empirical medicine, Mongolian medicine has valuable medical wealth left by many predecessors. However, many Mongolian medicine physicians and scholars do not study the thinking modes and reasoning methods in Mongolian medicine [31]. The Mongolian medicine physicians utilize the three diagnostic methods of "look, question and feel the pulse" in combination with the above reasoning methods for comprehensive analyses instead of using a certain reasoning method alone and focus on patients for verification. These reasoning methods are used in overall observation of patients and judgment of local diseased regions for correct diagnoses. They do not directly use drugs for certain disease during treatment. They will observe during treatment thus combining recognition and practice and treating diseases radically [32].

The recognition and practice processes of diseases in Mongolian medicine not only have their own characteristics but also raise the recognition and practice to a higher level under the idea of microscopic balance. One typical example is cerebral concussion-a peculiar therapy in Mongolian medicine. The cerebral concussion in Mongolian medicine is acquired by the nomadic people's observation and cognition of the animals and livestock during daily hunting and pasturing. Anyone who lives in a pasturing area knows that livestock must be subjected to concussion for alleviation or recovery when concussion occurs. Such a universal phenomenon in nature inspires the predecessors in Mongolian medicine. They propose the ideology of "treating concussion with concussion, combination of concussion and rest, and first concussion and then rest" based on their unique understanding [33]. Most people will choose to convalesce when their heads are shocked and impacted whereas the patients are required to act in a diametrically opposite way in Mongolian medicine. The predecessors have invented the cerebral concussion therapy in Mongolian medicine by combining their thorough understanding of concussion and practice. The normal position of the internal cerebral tissue will shift thus blocking the systemic blood circulation and causing pathological changes of "stagnation leading to pain". The patients' conditions will exacerbate if no timely treatment is administered. Thus, the blood circulation should be recovered on the basis of the "treating concussion with concussion". It also embodies the ideology of macroscopic balance and unity. Smooth systemic blood circulation can make the patients recover.

\section{Conclusion}

Substantial theoretical basis, clinical experience, and reasoning thinking accumulated in production and lives over thousands of years are integrated in Mongolian medicine. The original perceptual knowledge is gradually being raised to a higher level of conceptual knowledge. It plays a significant role in preventing, diagnosing, and treating disease. It better makes great contribution to reproduction of Mongol nationality.

Of course, the above-mentioned innovation involves ingenious combination of the ethnic medicine and logic. We propose our own opinions by reference to the research on medical logic and logical thinking in traditional Chinese medicine and on the basis of our plain understanding of Mongolian medicine and logic. We analyze and study the unique aspects of reasoning in Mongolian medicine, the systems of thinking and reasoning in Mongolian medicine and the regularities of logic in Mongolian medicine [35]. The logic is extensive and profound. The defined thinking modes and the concluded reasoning methods in Mongolian medicine are expected to further discussed. 


\section{References}

[1] Agula Bo. Mongolian Medicine and Pharmacy. Inner Mongolia Education Press. Huhhot. 2010: 1-2.

[2] Taibao Qi, et al. Diagnosis and Treatment of Mongolian Medicine and Its Clinical Application. Xinjiang Science and Technology Press. Urumqi. 2003.

[3] Sigen Tuo, Wuliji Tu, Minghui Xiong. Discussion on Mongolian Medicine Reasoning Methods. Journal of Medicine \& Pharmacy of Chinese Minorities.2014, 02: 60-63.

[4] Wenjun Sun. The research on the TCM diagnostic reasoning model and influencing factors. Shandong University of Traditional Chinese Medicine, 2012. (Doctoral Dissertation).

[5] Zhaorigetu Bao, Fenglan Bao. Comparative Study on Herbal Medidnes Shared byTraditional Chinese Medicine and Traditional Ethical Medicines. World Science and Technology/Modernization of Traditional Chinese Medicine and Materia Medica, 2006, 02: 65-67.

[6] Lixue Zhang, Donghao Wang. Righteous Features and Application of Abduction. Journal of Nanchang Hangkong University (Social Sciences).2013, 02: 1-7.

[7] Agula Bo. Mongolian traditional therapy. Inner Mongolia Education Press. Huhhot. 2012.

[8] Perez G, Slippers B, Wingfield BD, Hunter GC, Wingfield MJ. Micro- and macrospatial scale analyses illustrates mixed mating strategies and extensive geneflow in populations of an invasive haploid pathogen. Mol Ecol 2010, 19: 1801-1813.

[9] Xiaohua Wang, Long Bao. Similarities and Differences on Cause of Disease between Traditional Mongolian Medicine and Traditional Chinese Medicine. Acta Chinese Medicine and Pharmacology. 2013, 05: 4-7.

[10] Xiangjun Zhou, Zhaorigetu Bao, Fenglan Bao. Comparative Study of Prescription Usage of TCM and TMEM in Treatment of Cerebral Concussion. Journal of Yurmml College of Traditional Chinese Medicine. 2007, 01: 20-22.

[11] Jigmed B. Survey on Mongolian Medical Science History. Journal of Medicine \& Pharmacy of Chinese Minorities. 2007, 01: $1-5$.

[12] Qiqige Wuren. Medical Anthropological Interpretation of Mongolian Shamanism. Central University for Nationalities. 2006. (Doctoral Dissertation).

[13] Hasibagen Se, Shulan Zhang. Long life: Mongolian medicine. Guangxi Normal University Press. Guilin. 2008.

[14] Fukai Huang. An Analysis of the Similarities and Differences of Pulse Diagnosis between Traditional Chinese Medicine and Mongolian Medicine. Chinese Tibetology. 1999, 02: 130-134.

[15] Bater Harlun. Mongolian bone injury. Inner Mongolia People's Publishing House. Huhhot. 2010.

[16] Taogao Han. Legend of Kerqin Bao'family in Mongolian orthopedic. Inner Mongolia People's Publishing House. Huhhot. 2005.

[17] Cooper A. A Case of Aneurism of the Carotid Artery. Med Chir Trans 1809, 1: 1-12 11
[18] Andri M, Kyriakidou O. Professional autonomy under pressure: towards a dialectical approach. J Health Organ Manag 2014, 28 : 635-652.

[19] Xueli Liu. On the Historical Development of Medical Philosophy. ACTA Universitatis Medicinalis Nanjing (Social Science). 2002, 04: 263-267.

[20] Rong Chen, Yunfei Jiang, Li Lin. Stuty of Abductive Reasoning State of the Art and Problems. Computer Science, 2003, 05: 23-25.

[21] Jiang Qiu, Qinglin Zhang. A Review of Researches on Reasoning. Journal of Ningbo University (Education Science). 2003, 02: 5-8.

[22] Rodon J, Perez J, Kurzrock R. Combining targeted therapies: practical issues to consider at the bench and bedside. Oncologist 2010, 15: 37-50.

[23] Genyuan Su, Haiqing Huang. Similarities and Differences of "Three Diagnoses" in Mongolian and Chinese Medicine. Journal of Medicine \& Pharmacy of Chinese Minorities. 2002, 04: 49.

[24] Jialu Fan. A Probe into the Characteristics of Medical Logic. Medicine and Philosophy. 2002, 04: 53-55.

[25] Zhang W, Li X, He H, Wang X. Identifying network public opinion leaders based on Markov Logic Networks. Scientific World Journal 2014, 2014: 268592.

[26] Breivik J. [Darwinian logic-provocation and resource in medical thinking]. Tidsskr Nor Laegeforen 2002, 122: 2809-2811.

[27] Chiang HC, Chang HH, Huang PY, Hsu M. On the qi deficiency in traditional Chinese medicine. Taiwan J Obstet Gynecol 2014, 53: 317-323.

[28] Baisheng Jiang, Hong Liu. Differences between medical thinking and legal thinking. Medicine and Philosophy. 2008,01:29-31.

[29] Yingxiu Luo. A Probe into Pierce's Abductive Reasoning. Journal of Inner Mongolia Agricultural University (Social Science Edition).2007, 05: 239-241.

[30] Miranda GH, Felipe JC. Computer-aided diagnosis system based on fuzzy logic for breast cancer categorization. Comput Biol Med 2015, 64: 334-346.

[31] Rhee J, Nejad TM, Comets O, Flannery S, Gulsoy EB, Iannaccone $\mathrm{P}$, et al. Promoting convergence: the Phi spiral in abduction of mouse corneal behaviors. Complexity 2015, 20: 22-38.

[32] Meng QB, Yin YX, Zhang DZ, Yang GP. [Study on syndrome quantification, differentiation and classification of traditional Chinese medicine with data envelopment analysis]. Zhongguo Zhong Yao Za Zhi 2013, 38: 1631-1642.

[33] Zhou J, Zhu J, Chen M, Jiang M, Zhang Z, Zhan Z, et al. Logical thinking in pattern differentiation of traditional Chinese medicine. J Tradit Chin Med 2013, 33: 137-140.

[34] Anastasi JK, Currie LM, Kim GH. Understanding diagnostic reasoning in TCM practice: tongue diagnosis. Altern Ther Health Med 2009, 15: 18-28.

[35] Zhang H, Zhu X. [TCM diagnosis and reasoning method based on grey relational analysis]. Sheng Wu Yi Xue Gong Cheng Xue Za Zhi 2007, 24: 206-209. 